\title{
Application of soft-open points for the interconnection of neighbouring low voltage distribution networks Patrick Favre-Perrod ${ }^{1 *}$, Enea Auberson ${ }^{1}$, Cédric Bernasconi ${ }^{1}$, Andéol Demierre ${ }^{1}$, Serge Gavin ${ }^{2}$, Mauro Carpita ${ }^{2}$, Arnoud Bifrare ${ }^{3}$
}

\author{
${ }^{1}$ University of applied sciences and arts western Switzerland, Bd. de Pérolles 80, Fribourg, Switzerland \\ ${ }^{2}$ University of applied sciences and arts western Switzerland, Rte de Chesaux 1, Yverdon-les-Bains, Switzerland \\ ${ }^{3}$ Romande Energie, Rue de Lausanne 53, Morges, Switzerland \\ *patrick.favre-perrod@hefr.ch
}

Keywords: Power electronics, Decentralized generation, Voltage control, Active networks, Soft open point

\begin{abstract}
The use of a soft-open point (SOP) to interlink two LV networks in order to increase the PV hosting capacity has been investigated in a study preparing a pilot demonstration project within Romande Energie's (DSO in western Switzerland) grid. The contribution describes the selection of the location, the control mode and the size of the demonstrator based on the achievable improvement of the network performance in terms of voltage variations, cable / transformer loading and network losses. The size of the SOP has also been compared to the achievable distributed generation hosting capacity increase. The interface and protection of the SOP into the network is implemented using both local protection criteria and transfer tripping over a radio link. Simulations confirm that the chosen location and control mode for the SOP permit to achieve a more balanced utilisation of the available network capacity. Overall, more PV can be added to the network with no adverse effect on voltage quality or on the system's fault behaviour. On this basis, a demonstration SOP has been developed and tested in a laboratory environment. The final step has been to deploy the device into the distribution grid.
\end{abstract}

\section{Introduction}

Soft-open points (SOP) are two-port power electronic converters with the aim to control power flows within distribution networks, e.g. in normally radial or open-loop MV and LV systems. The SOP establishes a controlled connection between two parts of the grid normally not connected: the control system of the converter can establish arbitrary active power flows and reactive power infeeds. Several objectives can be pursued, including better repartition of loads on lines and transformers, reduced voltage variations, reduced losses, etc. In the context of the installation of distributed renewable energy sources (RES), this usually implies that an increase of the hosting capacity for distributed (PV) generation is possible $[1,2]$. This increase can be achieved without reinforcing the grid by traditional means, i.e. upgrading cables and transformers.

This contribution describes the placement, the control and the protection for a normally closed SOP to be deployed into Romande Energie's LV distribution system. The studies have been used to specify and develop a $50 \mathrm{kVA}$ demonstration device.

\section{SOP placement and control}

\subsection{Evaluation of the grid performance improvement}

To determine whether and how the effect of an SOP is positive on a low voltage network, four indices are used and combined into a score, which can be compared for several situations, e.g. different placements or control modes of the SOP. The indices used are defined in a manner that a low score is desirable:

- Voltage index: average quadratic voltage variation (for different load/generation situations) at all network nodes.

- Current index: average maximum relative loading of cables and lines.

- Transformer loading index: maximum relative transformer loading of all transformers considered.

- Losses index: weighted average of the relative network losses.

Each index is calculated for four typical load/generation snapshots and two extrapolated snapshots with an increased (future) distributed generation penetration. For the comparison of the effectiveness of an SOP, the following benchmark situations will be used:

- The reference case is the "initial situation", i.e. the current network without an SOP or any other modification. The addition of the SOP has to represent an improvement in comparison to this case in order to be a desirable solution.

- The optimal case is an SOP which is operated based on the result of an OPF. This case will not be achieved in practice, e.g. due to the limited amount of measurements available to the SOP control system.

- The coupling of two LV networks with a cable is another interesting comparison case. It will 
illustrate the benefit of controlling the load flows and reactive power infeeds.

\subsection{Control strategy for the SOP}

For the demonstration in LV networks, the requirements on the control and protection systems were to be independent of external real-time measurements (as such systems are rarely deployed), to provide its own communication links (fast internet cannot be assumed to be available) and simplicity (in view of daily operation). An optional interface to other measurement systems was however added, which allows an improved control if such external systems are available. Three control modes have been selected for a study and implemented into the SOP converter for on-site testing. The objectives of these three control modes were respectively:

- TRABA mode: to equalize the transformer relative loadings. The active power (essentially) through the SOP is adjusted until the relative loading of the distribution transformers feeding the two connected LV networks are identical or the SOP reaches its maximum apparent power.

- VOLTSAME mode: to reduce the voltage difference between the two networks. The apparent power at both ports of the SOP is adjusted until the voltage magnitude at both ports of the SOP is the same (or the SOP reaches its limits).

- VSTB mode: to combine the above two objectives.

The effects of the control modes on the voltage variations during operation, the maximum loading of cables and transformers as well as the network losses (including the SOP) can be evaluated with the score system introduced in section 2.1. For the choice of the SOP control mode and location, the emphasis is on the relative value of each possible choice: the ranking of the solutions is more relevant than the absolute score. The increase of the RES hosting capacity is indeed a quantitative criterion that will be discussed in chapter 3 .

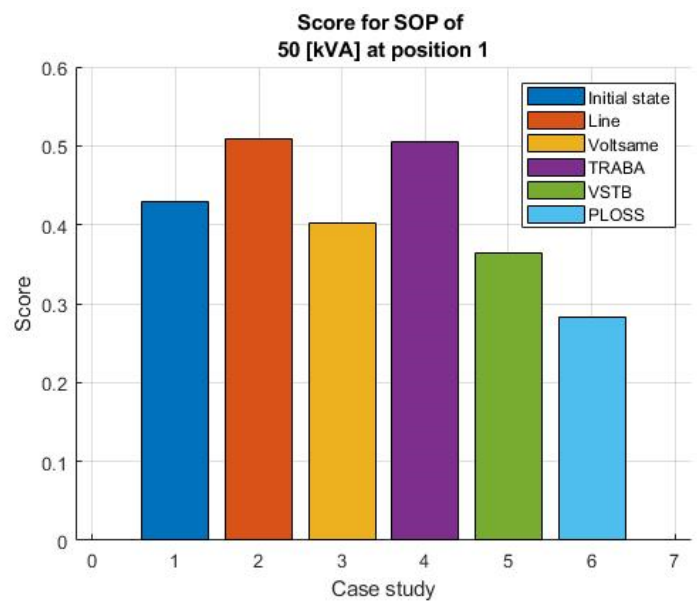

Figure 1: Comparison of grid control strategies (Voltsame: minimise voltage difference; TRABA: balance transformer loading, VSTB: Voltsame + TRABA, PLOSS: OPF).
Each of the control modes has been evaluated with the four indices previously described. The results for the three SOP control modes are shown in the center of Figure 1. The lower the score shown in Figure 1 is, the better the investigated variant is. Two benchmarks are used for comparison: i) the current situation without an SOP ("initial state") and ii) the addition of an ideal line instead of the SOP ("line"). The addition of the SOP would improve the voltage and loading situation, whereas the VSTB strategy is the most promising in this case. For further comparison, the result of an OPF with an objective to minimize network losses (including the SOP) is shown with the label "PLOSS".

The initial conclusion is that a galvanic coupling of the two distribution networks is not desirable, due to adverse effects on loading and losses. The OPF result on the right of Figure 1 is an indication of an ideally achievable improvement. The condition is however the availability of all currents and voltages within the LV network to the SOP controller. Protection and communication of the SOP will be discussed in section 4.1 .

\subsection{Placement of the SOP}

Figure 2 shows the LV network selected for the demonstration. It is fed by two $\mathrm{MV} / \mathrm{LV}$ transformer stations with a $250 \mathrm{kVA} \mathrm{MV} / \mathrm{LV}$ transformer each. Several candidate locations for an SOP interconnecting the two LV networks are indicated by red digits in Figure 2. The candidate locations correspond to places where the two LV networks are closest to each other.

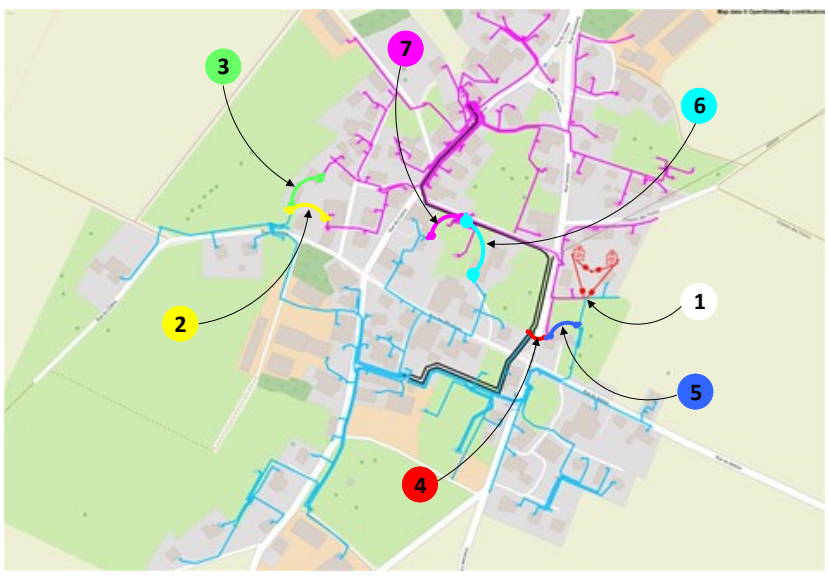

Figure 2: Considered candidate locations for an SOP in Chappelle-sur-Moudon.

The possible locations for the SOP have been compared against each other with the performance evaluation scheme introduced in section 2.1. Six extreme load / generation situations were considered. Figure 3 shows the score variation compared to the current situation with no SOP for the VSTB control strategy and an SOP size of $50 \mathrm{kVA}$. A positive score difference indicates an improvement, thus only an SOP placed at location number 1 would improve the current situation with the selected performance 
evaluation. For other locations, the additional losses implied by the use of power electronic converters, the additional loading of some cables within the LV network and the voltage variations implied would make the use of an SOP unattractive in the current situation.

Two factors need to be additionally considered for a medium and long-term horizon: firstly, adding distributed generation might decrease the performance score anyway and the SOP could still be needed in order to accommodate more distributed generation. Secondly, in practice it might be attractive to reinforce (moderately) the LV networks in combination with the SOP installation. These factors might increase the attractiveness of further locations for the SOP, with no impact on the ranking of the SOP locations determined here.

In addition to the technical criterion, planning permission, land owner approval and the feasibility from the perspective of civil engineering also need to be established. For the considered case study, location number 1 could be confirmed.

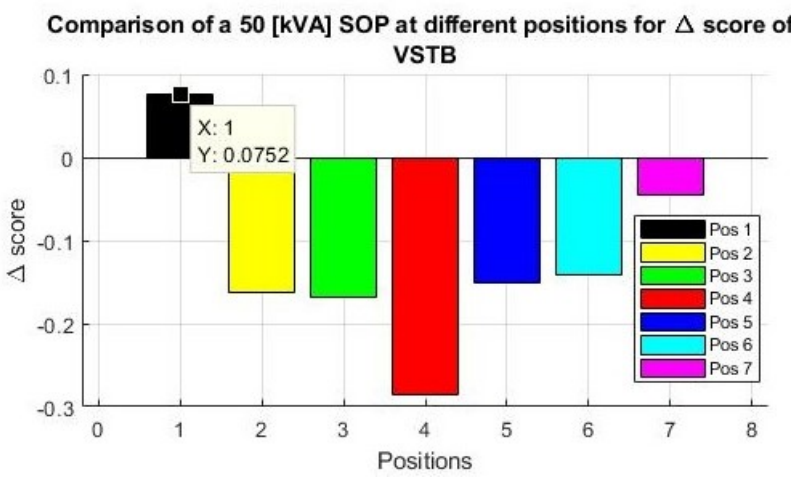

Figure 3: Achievable improvement of the network performance for different locations for the SOP.

\section{Improvement of the RES hosting capacity and sizing of the SOP}

Increasing the hosting capacity for RES is the main reason for considering the use of an SOP in this study. This criterion will be used in order to review the sizing of the SOP. Figure 4 shows the amount of distributed generation that could be added cumulatively to both LV networks considered, depending on the rating of the SOP installed at the location previously identified.

The blue bar indicates that a small amount of PV could be connected without the need to add an SOP. The next increments in SOP size from 50 to $150 \mathrm{kVA}$ roughly permit to install $50 \mathrm{kVA}$ of $\mathrm{PV}$ for each $25 \mathrm{kVA}$ of additional SOP capacity. The combined benefit of controlling load flows and local voltages permit this increase in hosting capacity which exceeds the added SOP throughput power. The increase of the SOP capacity to more than $150 \mathrm{kVA}$ does not increase the hosting capacity, since other limits (cable and transformer ratings e.g.) prevent any further incremental increase in hosting capacity. For the experimental setup in this project, a 50 $\mathrm{kVA}$ prototype was selected since during the relatively short demonstration time of 2 years, it is unlikely to expect the addition of more than $50 \mathrm{kVA}$ of solar power in the considered area.

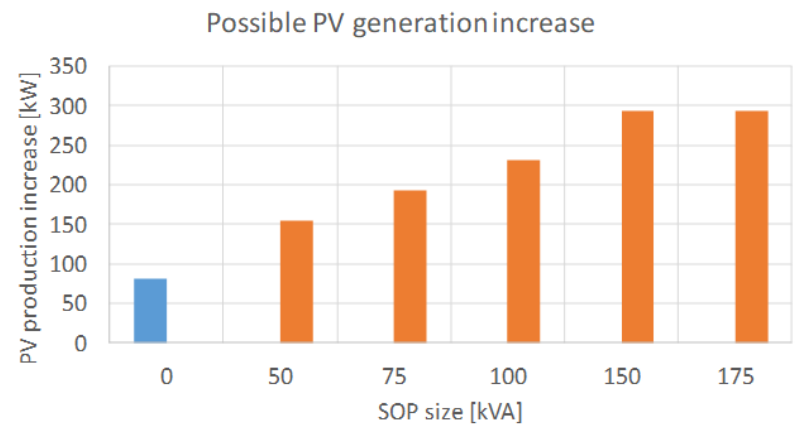

Figure 4: Improvement of the PV hosting capacity with an SOP.

\section{SOP deployment}

\subsection{Protection, communication and control system}

As discussed in [3], a potential advantage of the SOP is its compatibility with the already installed protection devices or fuses. This is achieved based on the comparatively fast action of power electronic converters compared to the protection of $\mathrm{MV}$ and LV distribution systems. The necessary precondition for this is to detect any fault or other situation requiring the SOP power flow to be interrupted and the SOP to be deactivated. For the experimental $50 \mathrm{kVA}$ SOP, a combination of local protection criteria and transfer tripping was selected:

- Local criteria: $\mathrm{U}>, \mathrm{U}<, \mathrm{f}<, \mathrm{f}>$ and $\mathrm{I}>$ relays on both ports of the SOP with settings inspired from the requirements made to distributed generators [4].

- Transfer tripping: $\mathrm{I}>$ and $\mathrm{U}<$ relays on the $\mathrm{LV}$ side of both $\mathrm{MV} / \mathrm{LV}$ transformers with a trip transfer to the SOP.

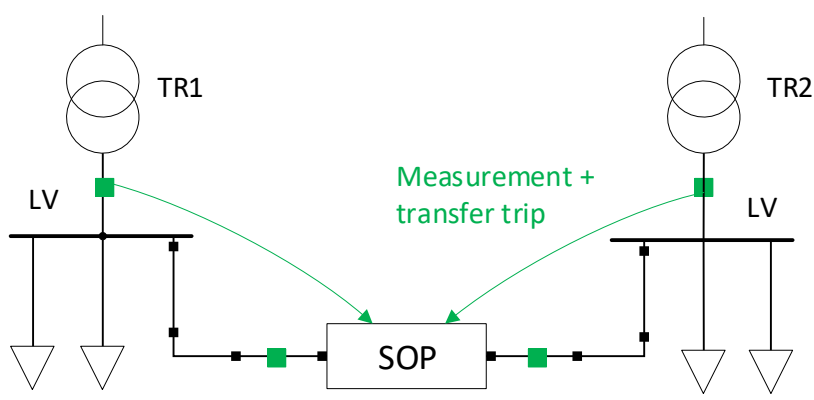

Figure 5: Communication scheme used for SOP protection.

The communication between the transformer stations and the SOP has been implemented using a dedicated radio link. Since the protocols used are based on IP (IEC 61850), a wired network (e.g. using fiber optic) can be used where 
it is available. As shown in Figure 5, the communication link is also used in order to acquire the measurements needed for determining the set point of the SOP depending on the control strategy as discussed above.

\subsection{Power electronic converter development}

A $50 \mathrm{kV}$ SOP has been developed and assembled based on a laboratory prototype described in [5-6]. The SOP basically is a back-to-back AC-DC-AC converter with a neutral controller. The control strategy for the converters is such that both converters are controlling the reactive power on their AC side, one converter is controlling the DC voltage and the other converter is controlling the active power transfer. The SOP converters and its network interfacing equipment are shown in Figure 6.

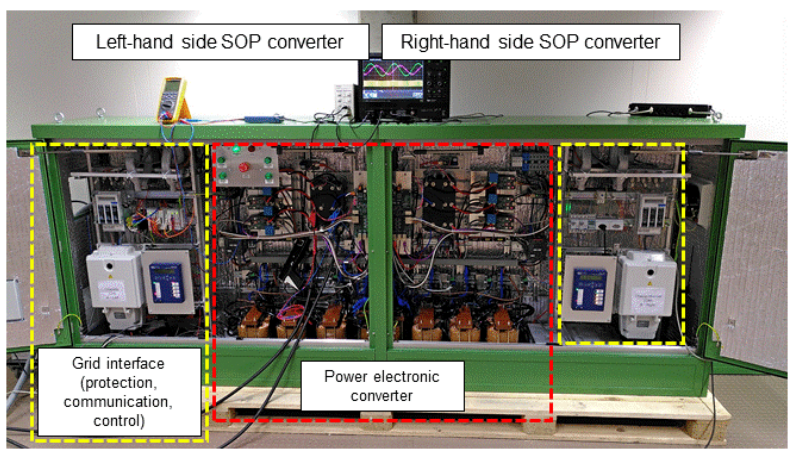

Figure 6: $50 \mathrm{kVA}$ SOP during laboratory testing.

\subsection{SOP impact on the test LV network}

In preparation for the SOP deployment to the real distribution network, the operation of the SOP has been simulated for several typical daily profiles and PV penetration rates. These simulations allow to verify that the choices for the location, the control mode and the sizing discussed earlier will allow the SOP to operate as expected. The simulations are performed using synthetic load curves that have been scaled in order to correspond to energy measurements available to the DSO.

A first simulation case shown in Figure 7(a) is a summer day with no PV injection, i.e. a light loading of the network. The simulations show that:

i. the SOP is operating as expected, i.e. it allows to equalize the p.u. loading of both transformers. This can be seen by comparing the transformer loadings with and without SOP.

ii. the loading of the SOP in this situation remains very low.

The more challenging situation of a summer day with maximum PV generation is shown in Figure $7(\mathrm{~b})$. The simulations reveal that:

i. the SOP can equalize the transformer loadings until it reaches its maximum power. Beyond this point, the loading of the transformers is different, although the difference is reduced by the SOP. ii. the SOP permits more PV generation since the loading with the SOP is within the possible range of both transformers. This is not the case without the SOP. In fact, this simulation shows a PV penetration that has been increased compared to the present situation in order to determine the limit (i.e. in this case $142.9 \%$ of the currently installed capacity).

iii. an arbitrage is needed between active and reactive power. In this example, the reactive power injection is limited to $10 \%$ of the SOP rating.

These simulations confirm the prospects to improve the situation within the considered LV networks and to increase its PV hosting capacity.

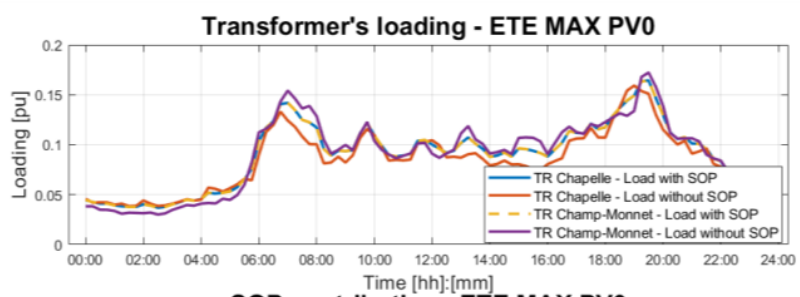

SOP contribution - ETE MAX PVO

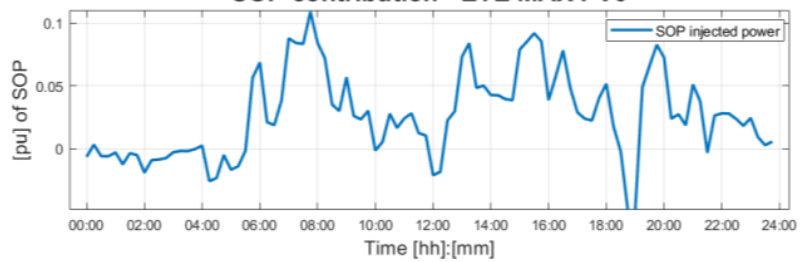

(a)
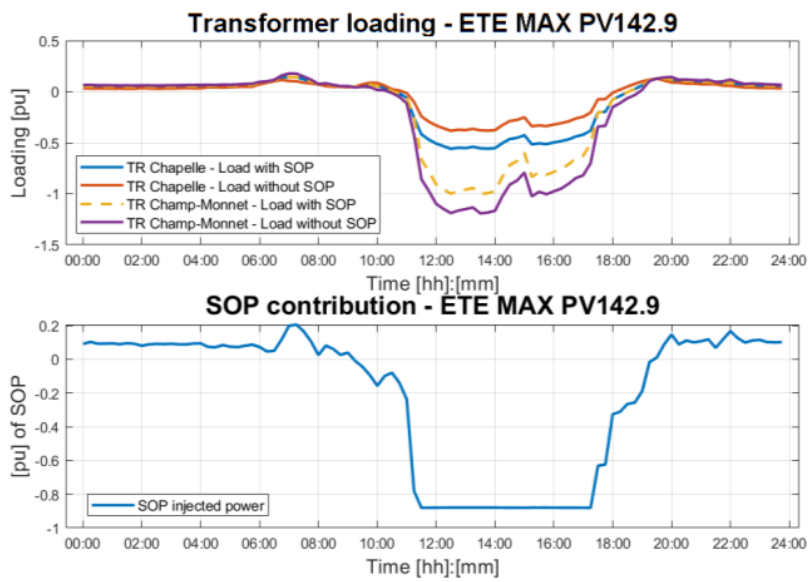

(b)

Figure 7: Transformer loading and SOP injected power for two extreme generation situations.

\section{Outlook}

The laboratory testing of the $50 \mathrm{kVA}$ SOP has been completed and the SOP is installed in the network. The controlled operation of the SOP is currently tested step-bystep, in order to identify any unwanted side-effects and validate its fully autonomous operation during the next project phase. 
Figure 8 shows measurements made when activating the VSTB control mode described in section 2.2. In Figure 8a, the active power at both transformer stations is shown together with the active and reactive power through the SOP (setpoints). As expected, the transformer loading is balanced by the activation of the VSTB control mode. Figure $8 \mathrm{~b}$ shows the voltages at the transformer stations and the SOP terminals. Controlling the reactive power infeed at both SOP terminals contributes to adjusting the voltages after activating the controlled mode and to reduce their variation over time.

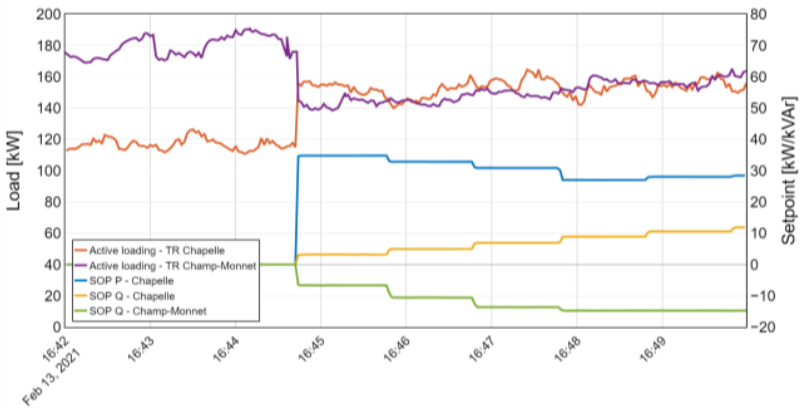

(a)

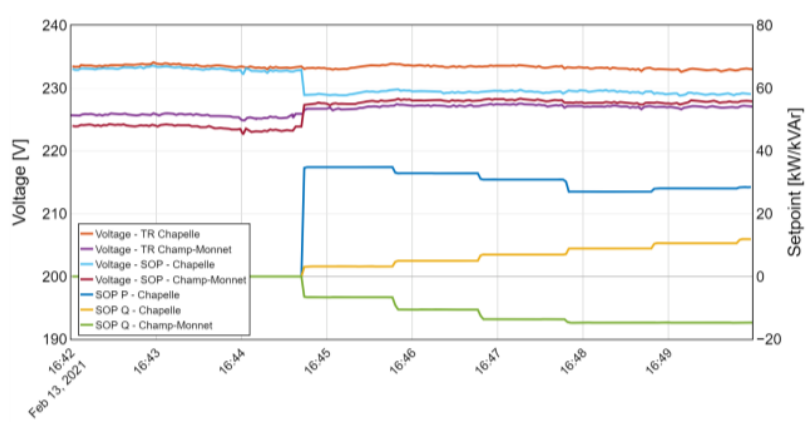

(b)

Figure 8: Power and voltage measurements before and after activating the controlled mode of the SOP.

Initial observations suggest that the effect of the transformer balancing is especially significant in case of high solar irradiance, since the installed PV systems are not distributed evenly across both LV networks. After initial testing is completed, The SOP's practical impact on the grid will be monitored in a pilot project for a planned duration of 18 months.

\section{Acknowledgements}

The work presented in this article is carried out within the frame of the Swiss Centre for Competence in Energy Research on the Future Swiss Electrical Infrastructure (SCCER-FURIES) with the financial and technical support of the Swiss Innovation Agency (Innosuisse - SCCER program), the Swiss Federal Office of Energy and Romande Energie.

\section{References}

[1] W. Cao, J. Wu, N. Jenkins, C. Wang \& T. Green, "Benefits analysis of Soft Open Points for electrical distribution network operation," Applied Energy, vol. 165, pp. 36-47, 3/1/ 2016.

[2] J. M. Bloemink \& T. C. Green, "Increasing distributed generation penetration using soft normally-open points," in IEEE PES General Meeting, 2010, pp. 1-8.

[3] P. Favre-Perrod, C. Dour, M. Allani, L. Eggenschwiler, A. Bifrare, M. Carpita, et al., "Softopen points for medium voltage networks - A case study," in 25th International Conference \& Exhibition on Electricity Distribution, Madrid, 2019.

[4] Swisssolar, Swissgrid \& VSE, "RR/IPE-CH," Réglages Suisse, Verband Schweizerischer Elektrizitästunternehmen, Aarau, 2017.

[5] T. Pidancier, M. Bozorg, D. Roggo, P. Favre-Perrod \& M. Carpita, "15 kVA Three-Phase Low-Voltage SOP Prototype Laboratory Tests Results," Energies, 2020.

[6] S. Gavin, G. Courteau, G. Grosjean, A. Bifrare, P. Favre-Perrod, M. Bozorg, M. Carpita, " Laboratory Tests results of a $50 \mathrm{kVA}$ Soft Open Point prototype," submitted to EPE-ECCE 2021, Gent ICC, Gand, Belgium, 6-10 sept. 2021. 